\title{
PRODUCTOS CON OPORTUNIDADES DE DESARROLLO EN CHILE:
}

CARBÓN VEGETAL. Daniel Soto $Y$ Janina Gysling. Ingenieros Forestales, Instituto Forestal, Chile. dsoto@infor.cl; jgysling@infor.cl Sede Metropolitana. Área de Economia y Mercado,

\section{RESUMEN}

El Sector Forestal chileno muestra un importante desarrollo basado en las plantaciones forestales y una fuerte industria derivada de estas, pero la producción, que hoy reporta más de 5 mil millones de dólares anuales por retorno de exportaciones, está en una muy alta proporción cimentada en commodities, como pulpa y madera aserrada, entre otros, que involucran una limitada agregación de valor y resultan muy sensibles a los vaivenes económicos internacionales.

El Instituto Forestal, en la búsqueda de alternativas de innovación y diversificación productiva en el ámbito forestal, está realizando permanentemente estudios sobre productos con oportunidades de desarrollo en el pais y con este objeto analiza diversos productos madereros y no madereros, sus posibilidades de desarrollo, sus mercados locales y externos, los volúmenes que se transan en el mundo y sus precios.

Recientemente se realizó un estudio sobre carbón vegetal, mucilagos de algarrobo (Prosopis chilensis) y pisos de madera, en el presente trabajo se presenta los resultados obtenidos para el carbón vegetal.

Palabras clave: Carbón vegetal, mercado, energía

\section{SUMMARY}

The Chilean Forestry Sector has an important development based on the planted forests and the strong associated industry, however the production, which currently reports over than 5 thousand million dollars in exports, is composed in a very high proportion by commodities, as pulp, sawn wood and other products, which involves a limited added value and are highly sensitive to changes in the world markets.

The Forest Institute, in searching innovative alternatives and productive diversification in the forest area, is carrying out studies on development opportunities with different products, analyzing wood and non wood forest products, its development possibilities, the domestic and international markets, the volumes sold in the world and the prices.

Recently it was made a study on charcoal, Prosopis mucilage (Prosopis chilensis) and wood floors, results on the first, charcoal, are presented in this paper.

Key words: Charcoal, market, energy. 


\section{INTRODUCCIÓN}

La diversificación de productos para la oferta nacional y exportable siempre es una necesidad en una economia como la chilena, basada principalmente en commodities. El sector forestal tiene un aporte cercano al $3 \%$ del Producto Interno Bruto (PIB) y de un $7 \%$ en las exportaciones nacionales, dentro de las cuales, la celulosa tiene la mayor participación (INFOR, 2007).

Si bien la proporción de los productos con mayor grado de elaboración en las exportaciones forestales de Chile se ha incrementado en los últimos años, la participación relativa de éstas se concentra en unos pocos productos, situación que por cierto es ventajosa para el pais, por el importante desarrollo de la industria asociada, pero a la vez se transforma en una desventaja debido a que la baja diversificación de productos dificulta la superación de las turbulencias negativas de los mercados, como así también la focalización en pocos mercados; la crisis financiera en Estados Unidos es un claro ejemplo de lo anterior. Por esto, disponer de una amplia variedad de bienes en la oferta de un pais permite compensar los efectos negativos de una reducción de las exportaciones, junto con mantener un mayor equilibrio en el largo plazo.

Bajo este contexto, el Ministerio de Agricultura ha encomendado al Instituto Forestal entregar antecedentes de algunos productos con posibilidades de desarrollo en Chile, como una manera de contribuir a esta diversificación. Para ello, se recopiló antecedentes generales del mercado mundial y local de algunos productos que se estima tienen buenas posibilidades de desarrollo. La selección se basó en las siguientes consideraciones: a) escaso desarrollo de productos que ya están presentes en el pais; b) nulas o minimas exportaciones; $c$ ) importaciones crecientes y d) diversos otros aspectos que permiten visualizar un desarrollo exitoso o que suscitan el interés en profundizar el estudio.

El carbón vegetal fue analizado por ser un producto de precario desarrollo en el pais, aún cuando ha estado por decenas de años presente en la economia nacional, incluso con grandes volúmenes de producción anual. Sin embargo, existen importantes falencias en la calidad del producto, fundamentalmente porque está asociado a la pequeña agricultura campesina quienes no utilizan técnicas apropiadas de carbonización, no tienen dedicación exclusiva para desarrollar esta actividad y se insertan en un mercado extremadamente informal. Una mejoria en estas condiciones, podria efectivamente elevar a este producto hacia el grupo de productos exportables.

El volumen de producción en Chile, cercano a las 250 mil toneladas por año (FAO, 2008), se consume enteramente en el pais, con casi nulas exportaciones. Las posibilidades de desarrollo apuntan a obtener un producto de probada calidad en encendido, contenido de humedad, tamaño, residuos, embalajes y otras características, que podria destinarse al mercado internacional, básicamente Europa, focalizándose en el segmento de las barbacoas. En efecto, cada vez existe menor disponibilidad de madera en ese continente y la elaboración de este tipo de productos se ve evidentemente afectada, por lo que se han incrementado las importaciones. El carbón vegetal en Europa es utilizado en un alto porcentaje para actividades recreativas, como consecuencia de la mayor demanda por tiempo libre. No obstante, conscientes del origen 
renovable de la materia prima, los consumidores exigen también que provenga de bosques manejados y con sellos que garanticen la calidad ambiental del producto.

Especial mención se hace al uso del carbón vegetal como materia prima para la generación de energia, aspecto que debe ser estudiado e investigado con profundidad, puesto que presenta un interesante potencial. Brasil ha sabido generar toda una industria del carbón vegetal asociada a la elaboración de hierro, manejando y plantando especies exclusivamente para estos fines. No obstante, persisten las quejas nacionales e internacionales sobre el uso indiscriminado de la selva amazónica para elaborar carbón.

\section{ANTECEDENTES DEL MERCADO MUNDIAL DE CARBÓN VEGETAL}

\section{Aspectos Generales}

De acuerdo con cifras de FAO, la producción mundial de carbón vegetal alcanzó en 2006 a los 43,5 millones de toneladas, cifra que en los últimos años ha mostrado una tasa de crecimiento moderada, en torno al $0,9 \%$ anual. No obstante, hoy se produce un $125 \%$ más de carbón vegetal en el mundo que en los años 70.

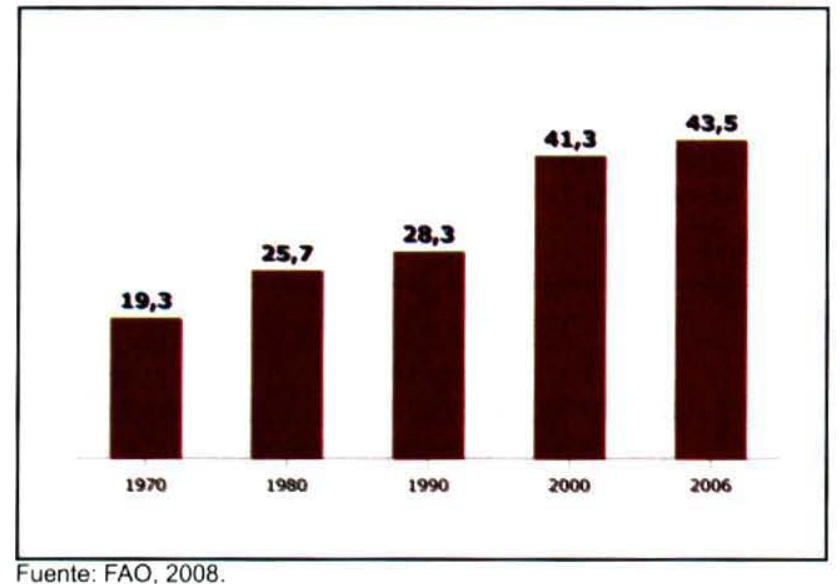

Figura $\mathrm{N}^{\circ} 1$

\section{PRODUCCIÓN MUNDIAL DE CARBÓN VEGETAL (Millones de toneladas)}

Según una encuesta realizada para el Programa de Asistencia para la Administración del Sector de la Energia del Banco Mundial, en 12 paises entre 1984 y 1993, el menor uso de leña y el ascenso de los productos del petróleo tienen una clara correlación con la elevación de los ingresos, asi como las nuevas politicas y los programas establecidos por los gobiernos. Sin embargo, existen regiones geográficas donde el consumo de energia se limita casi exclusivamente a la leña y el carbón vegetal, como muchos países de África. En este sentido, el ranking de paises productores de carbón vegetal muestra en las primeras ubicaciones a varios paises de este continente (Waddams, 2000). 
Destaca Brasil como el principal pais productor mundial, con una importante participación cercana al $25 \%$, incluso a principios de la presente década bordeando la tercera parte de la producción. Gran parte de esta producción está orientada a satisfacer la demanda de la industria siderúrgica brasileña.

La participación de Europa en el contexto mundial es marginal como zona productora, con apenas el 1,3\% del total, incluso Polonia, el mayor productor europeo tiene menos del $0,3 \%$ de incidencia. Otro gran productor, Estados Unidos, tiene un 2,1\% de participación mundial.

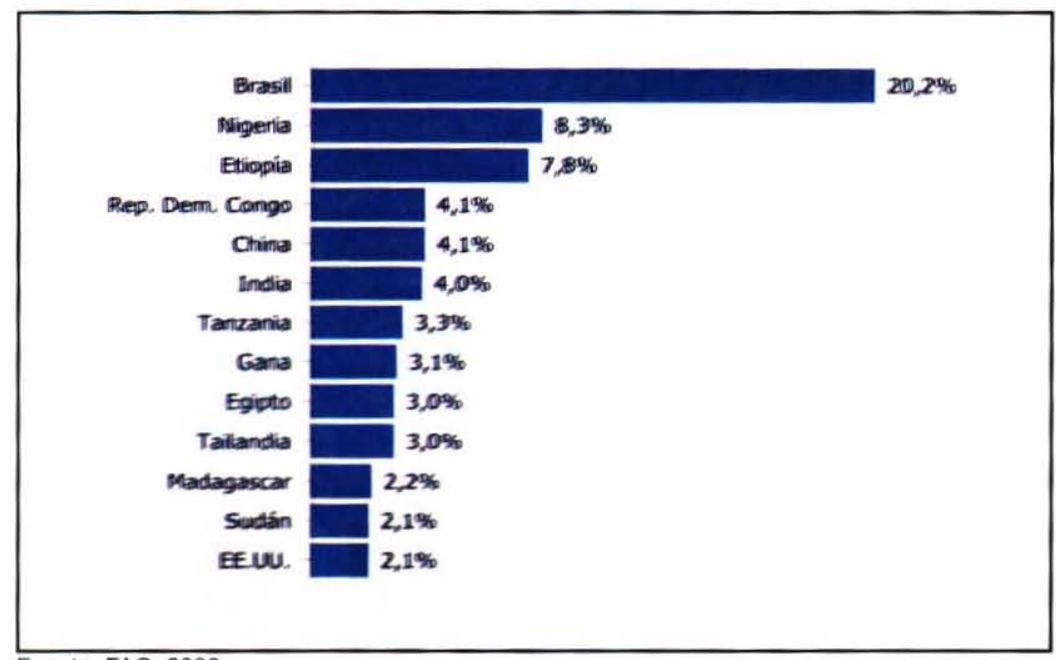

Fuente: FAO, 2008.

Figura $\mathrm{N}^{\circ} 2$

PRINCIPALES PAISES PRODUCTORES DE CARBÓN VEGETAL, 2006 (Total: $\mathbf{4 3 , 5}$ millones de toneladas)

\section{Características del Carbón Vegetal}

Carbón vegetal es definido como el residuo sólido que queda de carbonizar la madera, en condiciones controladas, en un espacio cerrado. Se obtiene de la combustión incompleta de la madera y sus características fisicas y quimicas dependen del tipo de madera usada y de las caracteristicas del proceso de carbonización.

La carbonización de la madera consiste en la transformación de dicha madera en carbón, bajo la sola influencia de la temperatura, siendo entonces una descomposición térmica en ausencia de aire, también llamada pirólisis. El control del proceso de la carbonización, se hace sobre la entrada de aire, para que la madera no se queme simplemente en cenizas, sino que se descomponga quimicamente para formar el carbón vegetal. Los métodos modernos de producción de carbón no permiten entradas de aire, favoreciendo el rendimiento, pues asi no se quema excesiva madera, mejorando la calidad. 
Los usos del carbón vegetal son múltiples y algunos datan de muchos siglos. Entre las aplicaciones domésticos del carbón vegetal están las culinarias y de calefacción y la utilización al aire libre en parrillas y barbacoas, mientras que el uso industrial se aprecia en actividades tales como el secado directo o indirecto de otros productos y en la utilización como combustible interno en la fabricación de cal y de cemento. Otros usos industriales son la separación de metales y trabajos de fundición, pigmentación de tintas de imprenta y pinturas, fuegos artificiales, plásticos, producción de caucho y otros.

El empleo de carbón vegetal se debe al hecho de ser un combustible proveniente de un recurso renovable, liviano, fácil de manipular y con valores calorificos comparables con el carbón mineral. Las principales características del carbón vegetal son:

a) Rendimiento en peso entre un $20 \%$ a $30 \%$ del peso seco de la madera

b) Rendimiento en volumen del $50 \%$ del volumen de madera

c) Peso especifico real $0,2-0,5 \mathrm{~g} / \mathrm{cm}^{3}$.

d) Peso específico aparente $1,3-1,5 \mathrm{~g} / \mathrm{cm}^{3}$.

e) Dureza variable según densidad de la madera.

f) Humedad del $1 \%$ al $16 \%$.

g) Hidrocarburos del $7 \%$ al $30 \%$.

h) Contenido fijo de carbono del $80 \%$ al $90 \%$.

\section{Cuadro $\mathrm{N}^{\circ} 1$ \\ PODER CALORÍFICO SUPERIOR DE ALGUNAS SUSTANCIAS}

\begin{tabular}{|c|c|}
\hline Sustancia & Kcal/kg \\
\hline Hidrógeno & 34.000 \\
\hline Gas natural & 10.900 \\
\hline Hulla (carbón mineral) & $5.900-8.200$ \\
\hline Carbón vegetal & $\mathbf{8 . 0 0 0}$ \\
\hline Alcohol etílico & 7.100 \\
\hline Papel de diario & 4.400 \\
\hline
\end{tabular}

(Fuente: INFOR,1987).

\section{Antecedentes del Mercado Internacional de Carbón Vegetal}

El comercio internacional de carbón vegetal y sus derivados, si bien es muy reducido en comparación con los grandes rubros forestales e industriales, constituye un nicho atractivo para muchos países productores de carbón vegetal de maderas duras, que tienen el potencial para ser interesantes proveedores a nivel internacional.

En muchos paises desarrollados, especialmente de Europa, y en Estados Unidos, el consumo doméstico de carbón vegetal y también de briquetas está asociado con la recreación. 
Este es un mercado en crecimiento por efecto del aumento en el ingreso familiar de las capas medias de la población en estos paises y por la mayor disponibilidad de tiempo libre. En algunos paises de la Unión Europea también contribuye a este fenómeno la menor disponibilidad de leña.

Las cifras de comercio internacional de carbón vegetal se encuentran clasificadas bajo el código armonizado 4402, cuya glosa es "carbón de madera, incluido el carbón de cáscaras o de huesos (carozos) de frutos, incluso aglomerado", la cual no presentaba subdivisiones de códigos hasta el año 2006. A partir del año 2007, luego de la actualización realizada a la Nomenclatura Internacional, se realizó una apertura a esta partida, para identificar al carbón vegetal de la especie bambú. De esta forma, se creo el código 4402.10.00 "De Bambú" y código 4402.90.00 "Las Demás". En todos los paises del mundo se utilizan estos dos códigos para identificar al carbón vegetal.

En el año 2006 el comercio exterior en el mundo movilizó entre 1,3 a 1,4 millones de toneladas de carbón vegetal, lo que equivale a menos del $3 \%$ de la producción mundial (FAO, 2008).

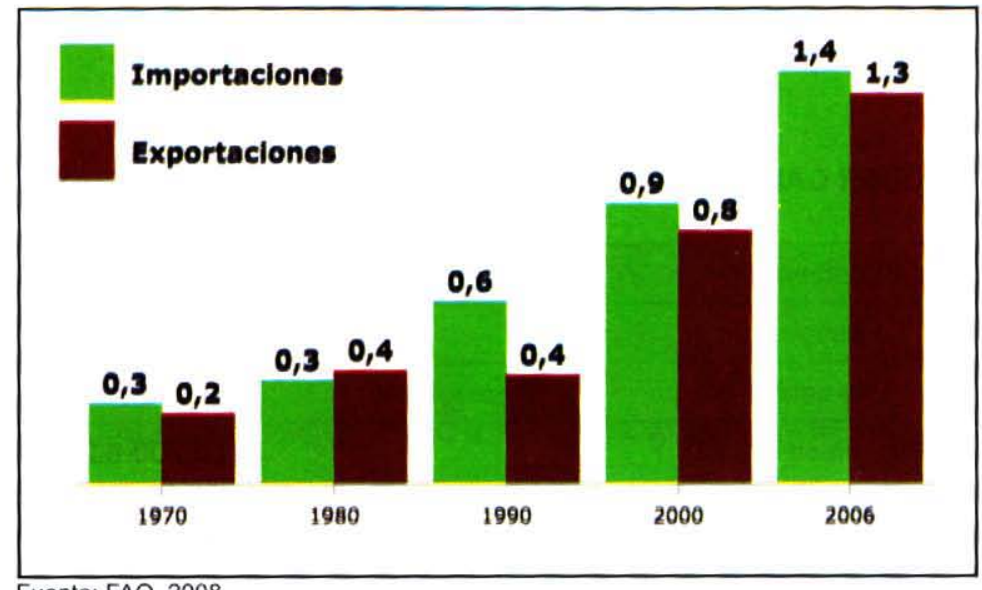

Fuente: FAO, 2008.

\section{Figura $\mathrm{N}^{\circ} 3$ COMERCIO MUNDIAL DE CARBÓN VEGETAL (Millones de toneladas)}

Los mayores compradores de carbón vegetal son Japón, Alemania, Corea del Sur y China. En la Figura $\mathrm{N}^{\circ} 4$ destacan varios países de la región europea dentro de los principales importadores mundiales, lo que advierte la importante potencialidad que ofrece este mercado como destino para el envío de carbón vegetal, principalmente por el nivel de desarrollo, asociado a un mayor ingreso de las personas, lo que sugiere una interesante demanda por tiempo libre y recreación. 
Japón también se perfila como un interesante mercado toda vez que el uso de este producto ha retomado fuerza en el consumidor común y corriente, asi como tambièn en algunas industrias que lo utilizan por sus múltiples beneficios y propiedades. Despues de un periodo de constante reducción en el consumo interno de carbón vegetal, hace unos años las importaciones comenzaron paulatinamente a crecer en este mercado y se espera una dinámica similar en el futuro.

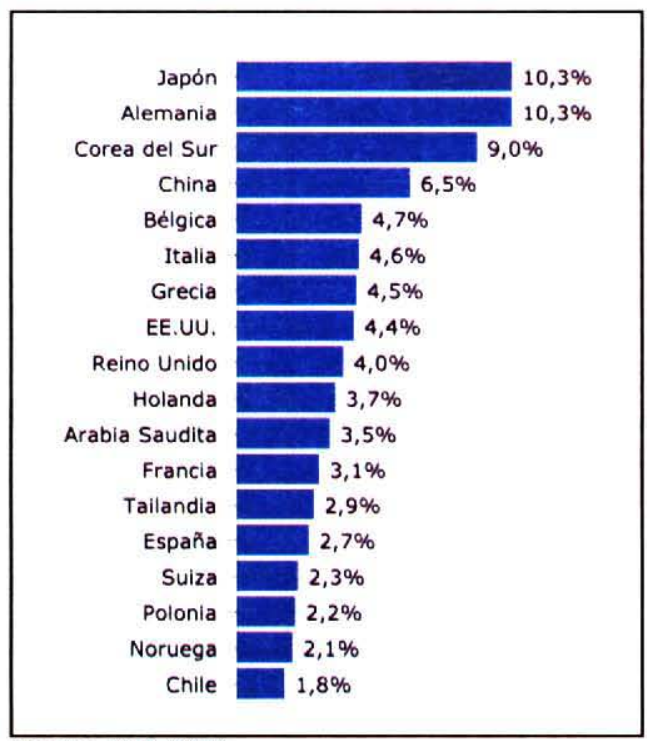

(Fuente: FAO, 2008).

\section{Figura $\mathrm{N}^{\circ} 4$ \\ PRINCIPALES PAISES IMPORTADORES DE CARBÓN VEGETAL, 2006} (Total: 1,4 millones de toneladas)

\section{- Europa}

La producción de carbón vegetal en toda la región europea ronda las 556 mil toneladas anuales, que equivale a solo el $1,3 \%$ de la producción mundial. Su uso se remonta a varios miles de años atrás y fue importante para muchas necesidades, como la fundición de metales y la obtención de pólvora.

A principios del siglo XVIII se mejoró el uso del carbón mineral para fundición de metales, que por lo demás era mucho más barato que el carbón vegetal, desplazando completamente a este último y lo relegó fundamentalmente a un uso doméstico. En la actualidad Polonia (130.000 mil t) y Eslovaquia (83.000 t) son los dos mayores productores europeos, que representan el $23 \%$ y $15 \%$ de la producción de carbón vegetal de la región. Sin embargo, en un contexto mundial, los paises de Europa tienen un rol marginal como productores. 
Esta baja producción de carbón en Europa hace que la demanda deba suplirse con importaciones. Estas compras en la actualidad se acercan a las $700.000 \mathrm{t}$ anuales, constituyèndose asi en uno de los mayores mercados internacionales para la exportación de carbón vegetal de uso doméstico. Los principales importadores europeos son Alemania, Bélgica, Italia, Grecia y Reino Unido; estos cinco paises se llevan el $54 \%$ de todas las importaciones europeas de carbón vegetal.

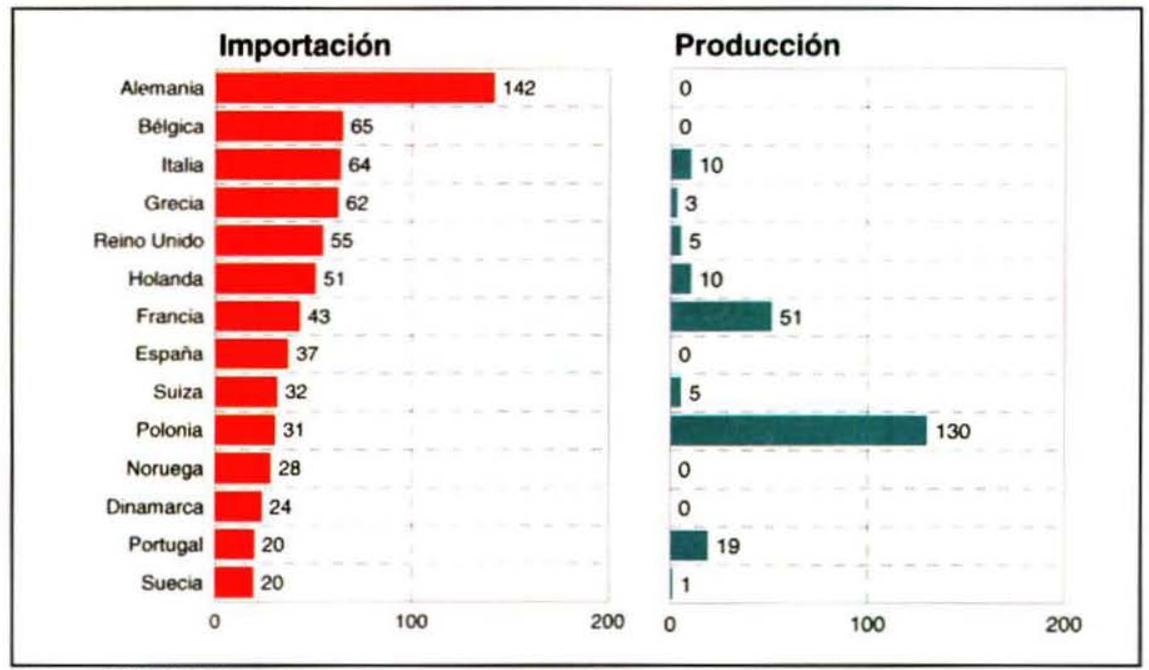

(Fuente: FAO, 2008).

\section{Figura $\mathrm{N}^{\circ} 5$ \\ PRINCIPALES IMPORTADORES EUROPEOS DE CARBÓN VEGETAL Y SU PRODUCCIÓN, 2006 (Miles de toneladas)}

En el Reino unido se consumen alrededor de 60 mil toneladas anuales de carbón para barbacoas, segmento de mercado que ha tomado importancia sólo en la última década. La mayor parte del carbón que se importa al Reino Unido proviene de Asia y, de acuerdo con los comercializadores locales, normalmente es tratado con productos quimicos y la calidad del encendido es inferior comparado con el carbón de producción nacional. Al respecto, y conscientes de la necesidad de protección de los bosques en el mundo, se ha instado por parte de productores locales ingleses a utilizar carbón con sellos de certificación.

Cabe resaltar al mercado italiano, especialmente interesante, puesto que no se trata de compras exteriores para uso interno en forma significativa, sino que generalmente actúa como pais de introducción para una posterior distribución al resto de la Unión Europea. No obstante, se ha iniciado una interesante demanda por parte de algunos sectores como las pizzerias, quienes aprecian el ahumado de sus productos con carbón vegetal de determinadas especies y caracteristicas. 


\section{Cuadro $\mathrm{N}^{\circ} 2$ \\ PRINCIPALES PAISES IMPORTADORES EUROPEOS DE CARBÓN VEGETAL (Miles de toneladas)}

\begin{tabular}{|c|c|c|c|c|c|c|c|}
\hline País & 2000 & 2001 & 2002 & 2003 & 2004 & 2005 & 2006 \\
\hline TOTAL EUROPA & 423,2 & 503,4 & 508,7 & 571,2 & 632,6 & 760,1 & 721,6 \\
\hline Alemana & 112,1 & 113,6 & 113,6 & 113,6 & 138,3 & 149,5 & 141,5 \\
\hline Bélgica & 26,0 & 25,7 & 35,6 & 63,4 & 64,0 & 60,1 & 64,6 \\
\hline Italia & 46,0 & 41,0 & 41,2 & 46,7 & 49,6 & 56,8 & 63.6 \\
\hline Grecia & 16,7 & 25,9 & 32,2 & 45,0 & 50,6 & 53,9 & 62,4 \\
\hline Reino Unido & 45,0 & 41,6 & 43,6 & 54,5 & 57,0 & 53,2 & 54,6 \\
\hline Holanda & 20,0 & 14,0 & 17.5 & 19,7 & 24,0 & 56,8 & 50,9 \\
\hline Francia & 27,7 & 31,1 & 26,5 & 42,2 & 49,2 & 53,4 & 42,9 \\
\hline España & 16,1 & 16,1 & 28,0 & 33,0 & 42,0 & 45,0 & 37,0 \\
\hline Suiza & 10,4 & 31,4 & 34,9 & 10,0 & 10,0 & 32,5 & 31,5 \\
\hline Polonia & 3,6 & 10,3 & 11,7 & 12,7 & 15,8 & 22,2 & 30,5 \\
\hline Noruega & 43,0 & 71,0 & 45,0 & 40,6 & 33,6 & 77,1 & 28,3 \\
\hline Dinamarca & 13,0 & 12,0 & 17,3 & 20,5 & 17,0 & 23,8 & 23,8 \\
\hline Portugal & 9,2 & 11,0 & 15,0 & 18,0 & 27,0 & 18,0 & 20,0 \\
\hline Suecia & 10,9 & 14,0 & 15,9 & 17,5 & 18,3 & 19,4 & 19,5 \\
\hline Otros Europa & 12,4 & 32,7 & 9,7 & 8.9 & 11,2 & 15,5 & 23,6 \\
\hline
\end{tabular}

(Fuente: FAO, 2008).

En la Unión Europea, una caracteristica sobresaliente es la preocupación de los consumidores por la sustentabilidad de la producción de carbón, por lo que la tendencia general es impulsar la preferencia por productos certificados por organismos internacionales. Los principales proveedores internacionales para Europa son Malasia e Indonesia, pero destacan también Sudáfrica y Paraguay, asi como otros paises asiáticos y africanos con disponibilidades de maderas latifoliadas duras, aunque en estos casos se trata más bien de importadores europeos que establecen sus operaciones en busca de costos más bajos.

\section{- Estados Unidos}

Constituye el mercado más importante, por ingreso, población y por la costumbre de la tradicional barbacoa. De acuerdo a las estadisticas de la National Barbecue Association, tres de cada cuatro viviendas unifamiliares suburbanas tienen uno o más tipos de parrillas, estimándose que se hacen 2.900 millones de asados anuales. También se venden 11,4 millones de parrillas de todo tipo por año, y es común que existan dos tipos de parrillas por hogar. De esta manera el $57 \%$ de los usuarios tiene una parrilla a gas y el $54 \%$ una parrilla a carbón. El $95 \%$ de los usuarios de carbón lo utiliza bajo la forma de briquetas, alcanzando el consumo 
anual unas 884 mil toneladas, calculándose el volumen del negocio en unos 600 millones de dólares anuales (SAGPyA, 2003).

En el año 2006, Estados Unidos internó 61,3 mil toneladas de carbón vegetal, equivalentes a US $\$ 23,6$ millones. Sin embargo, esta cifra no refleja el porcentaje que tienen las briquetas y el carbón sólido en la composición de estas importaciones que, de acuerdo con la bibliografia, el primero de éstos sería el producto más demandado. México es el principal abastecedor de carbón vegetal a Estados Unidos, con cerca del $42 \%$ del volumen importado, aunque en el transcurso de los años este pais ha ido perdiendo terreno frente al crecimiento notable de Argentina, China y Paraguay como paises abastecedores. En 1998, el carbón vegetal importado a Estados Unidos provenia casi en su totalidad de México.

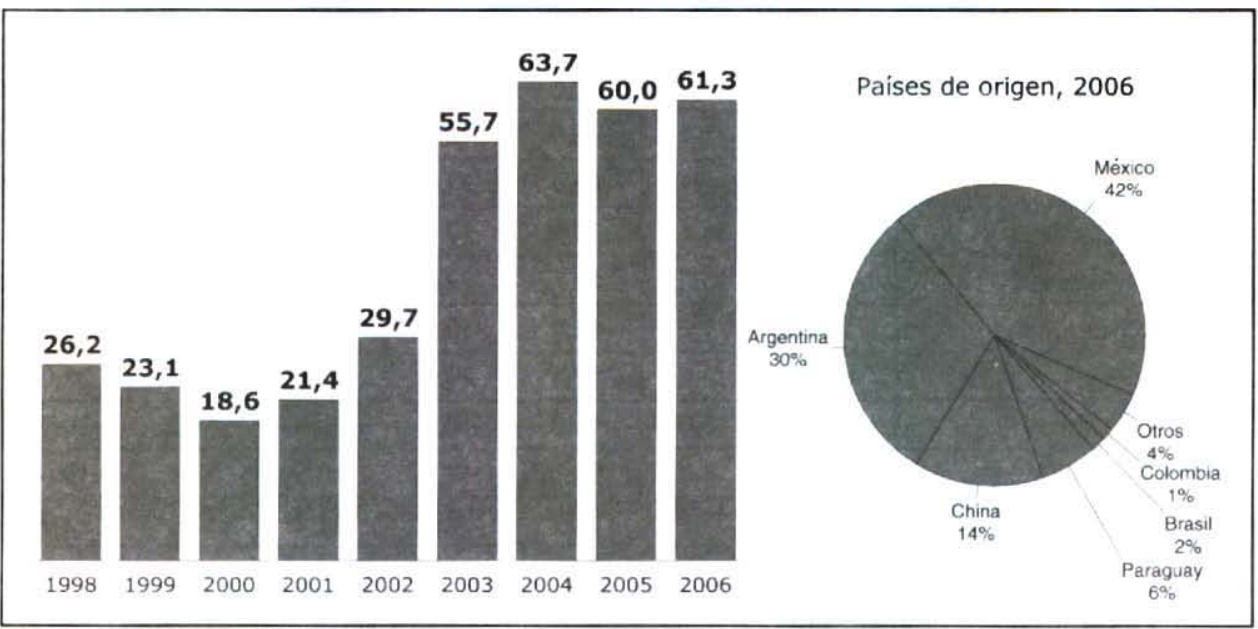

(Fuente: US Department of Agriculture HS 4402

\section{Figura $N^{\circ} 6$ \\ IMPORTACIONES DE CARBÓN VEGETAL EN ESTADOS UNIDOS (Miles de toneladas)}

Cabe destacar las importaciones de carbón vegetal de bambú (4402.10.00) que realizó Estados Unidos en 2007, las que sumaron 5.600 t. En estas compras, de los 6 primeros paises de origen (que aglutinan el $98 \%$ del volumen), 5 son latinoamericanos; el primero es Paraguay y le siguen Argentina, México, Colombia y Bolivia; entre ellos se ubicó Holanda. Ese antecedente debe ser considerado de sumo interés por parte de quienes están vinculados al recurso bambú en Chile.

Los leños artificiales, hechos con aserrin y aglutinantes constituyen un mercado más reducido. Las importaciones de este producto, en 1998 totalizaron 48 mil toneladas por un valor de US\$22,4 millones. Canadá es el principal proveedor con más del $97 \%$ del total, a lo que se suman pequeñas cantidades provenientes de China, Taiwán y Honduras. 
El carbón vegetal es considerado un producto de nicho (gourmet product), destinado a un grupo de consumidores más sofisticado que desea ahumar los alimentos. El $84 \%$ de los poseedores de parrillas son familias jóvenes o grandes de mayores ingresos y que normalmente viven en casas. Por este motivo, además, la oferta se concentra en carbones obtenidos de maderas aromáticas como robles, arces y frutales entre otras.

Existe una importante producción local en el mercado estadounidense, con más de 2.000 establecimientos registrados, basada en el aprovechamiento de la amplia base de especies latifoliadas. La leña, más que un sustituto parece comportarse como un producto de uso complementario por este segmento de los consumidores, destinado principalmente al ahumado de los alimentos.

El predominio del uso de briquetas y la existencia de la producción local hace que el mercado de los EEUU sea menos accesible para los productores de carbón que los mercados europeos.

\section{- Japón}

En Japón, el carbón vegetal es llamado "mokutan" o algunas veces "zumi". En el pasado, el carbón solia ser la principal fuente de combustible de uso doméstico y se le consideraba tan importante en la vida diaria como el mismo arroz. En la década del 50 la producción de carbón era de unas 2 millones de toneladas (FAO, 2008). Con el paso de los años y tras el advenimiento del gas y la electricidad de la mano del crecimiento económico, la producción cayó abruptamente, tal que después de 40 años apenas superaba las 50 mil toneladas anuales.

En los últimos años la producción ha caido significativamente en Japón, mientras que el consumo doméstico ha ido en crecimiento; por ello el origen del carbón vegetal que se utiliza en este pais es fundamentalmente importado. Las razones de ello radican en el envejecimiento de los productores locales de carbón y la falta de sucesores que lo continúen y también en la dificultad cada vez mayor para obtener las maderas apropiadas para carbonización en las zonas productoras tradicionales de Japón.

De acuerdo con FAO, la producción de carbón vegetal en Japón en el año 2006 fue de 33 mil toneladas, mientras que su comercio exterior movilizó $1.000 \mathrm{t}$ al exterior y 142 mil toneladas en importaciones, es decir, cerca del $82 \%$ de la demanda de carbón vegetal japonés proviene del exterior. Diez años atrás era el $60 \%$ (FAO, 2008). 


\section{Cuadro $\mathrm{N}^{\circ} 3$ \\ IMPORTACIONES DE CARBÓN VEGETAL EN JAPÓN}

\begin{tabular}{|c|c|c|}
\hline Año & $\begin{array}{c}\text { Toneladas } \\
\text { (miles) }\end{array}$ & $\begin{array}{c}\text { Monto } \\
\text { (US\$ millones) }\end{array}$ \\
\hline 1980 & 18,5 & 3,7 \\
\hline 1985 & 54,3 & 12,6 \\
\hline 1990 & 76,0 & 19,9 \\
\hline 1995 & 51,7 & 32,4 \\
\hline 2000 & 129,0 & 73,6 \\
\hline 2006 & 142,0 & 80,9 \\
\hline
\end{tabular}

(Fuente: FAO, 2008).

El carbón vegetal que llega a Japón proviene principalmente de China, Indonesia y Malasia, que representan cerca del $96 \%$ de las importaciones japonesas, cada uno con participaciones bastante similares. Otros mercados eventuales han sido Singapur y Tailandia.

Japón tiene una cultura milenaria en producción de carbón vegetal, con técnicas de elaboración que son admiradas en todo el mundo. Existes diversos tipos de carbón vegetal que en general se clasifican en dos grandes categorias: kuro-zumi (carbón vegetal negro) y shiro-zumi (carbón vegetal blanco). El carbón vegetal negro es suave y conserva la corteza, es fácil de encender y alcanza suficiente temperatura como para fundir metales y hacer trabajos de herreria (casi todo el carbón vegetal que se produce en el mundo pertenece a esta categoria). Para elaborarlo, se carboniza la madera a temperaturas entre 400 y 700 grados centigrados, después se sella el horno hasta que finaliza la combustión y la temperatura baja progresivamente.

Por su parte, el carbón vegetal blanco se hace quemando madera a temperaturas relativamente bajas durante algún tiempo y, casi al final del proceso, se eleva la temperatura del horno a unos 1.000 grados centigrados hasta poner la madera al rojo vivo. Posteriormente, se saca el carbón y se cubre de polvo para enfriarlo, que es una mezcla de tierra, arena y cenizas, dándole a la superficie del carbón un tono blanquecino. De ahí su nombre "carbón vegetal blanco." La rápida subida de la temperatura, seguida por un rápido enfriamiento, incinera la corteza y proporciona suavidad a su superficie.

Una parte importante del carbón importado a Japón es hecho de cáscaras de nueces, que se utiliza principalmente para hacer carbón activado. Otra parte es utilizada a nivel doméstico para actividades de recreación y tiempo libre (barbacoas) y en el creciente segmento de los servicios de alimentación. Una porción menor se utiliza para otros fines, por sus caracteristicas beneficiosas: Como absorbente de olores ambientales y sustancias dañinas, como generador de iones negativos, que se dice favorecen la tranquilidad mental y utiliza los efectos de infrarrojos. que se supone mejoran la circulación sanguinea. 
Investigadores en Japón han estudiado estos beneficios, explorando nuevos usos para desarrollar productos innovadores basados en carbón vegetal, como purificadores de agua (para beber y para baños), agentes conservadores de vegetales y otros alimentos frescos, reguladores de humedad para las paredes y pisos de casas y productos de aseo personal (desodorantes, jabones, etc.) (Kuniko, 2001).

\section{- Brasil}

Brasil es el mayor productor mundial de carbón vegetal de acuerdo con cifras de FAO (2008) y asi se ha mantenido por varios años. Por otro lado, es el mayor productor latinoamericano de acero, con una industria bastante madura y competitiva. La relación entre estos dos productos está en que la mayor parte de la producción del carbón vegetal brasileño se destina a la industria del acero. En efecto, para la obtención del acero se necesita pasar por varias etapas, la primera de ellas es la obtención de un material llamado arrabio, algunas veces denominado "hierro esponja", en el cual intervienen ciertas materias primas de las cuales el carbón (sea mineral o vegetal) cumple una función clave.

La historia mundial del acero señala que en a fines del siglo XIV se mejoraron algunas técnicas de producción, utilizando hornos de mayor tamaño para la fundición y se incrementó el tiro para forzar el paso de los gases de combustión por la carga o mezcla de materias primas. En estos hornos de mayor tamaño el mineral de hierro de la parte superior del horno se reducia a hierro metálico y a continuación absorbia más carbono como resultado de los gases que lo atravesaban. El producto de estos hornos era el llamado arrabio, una aleación que funde a una temperatura menor que el acero o el hierro forjado. El arrabio se refinaba después para fabricar acero (Enciclopedia Encarta, 2008).

La producción más típica de arrabio se realiza utilizando mineral de hierro, coque y caliza. El coque se quema como combustible para calentar el horno, y al arder libera monóxido de carbono, que se combina con los óxidos de hierro del mineral y los reduce a hierro metálico. Esta labor también se puede realizar con carbón vegetal. La caliza de la carga del horno se emplea como fuente adicional de monóxido de carbono y como sustancia fundente.

Prácticamente todas las plantas siderúrgicas de Brasil utilizan parte o la totalidad de carbón vegetal como materia prima para producir arrabio, producto que cumple con similares caracteristicas que el coque mineral. Muchas empresas comenzaron hace algunos años a extender sus negocios hacia el área forestal y otras del sector a potenciar la compra de terrenos para el cultivo de eucaliptos, los que se convertirán en carbón.

En favor y en contra del negocio del arrabio utilizando carbón vegetal se esgrimen básicamente razones medioambientales. En el primer caso se argumenta la capacidad de absorción de carbono que poseen los árboles, el cual será mucho más que el liberado en el proceso de fabricación del acero. Además, el coque despacharia a la atmósfera mucho más carbono que el carbón vegetal en la combustión; por cada tonelada de acero producido con coque se emiten a la atmósfera $5 \mathrm{t}$ de $\mathrm{CO}_{2}$. 
En el otro extremo, la discusión se centra en el origen del carbón vegetal que abastece a las plantas acereras. Numerosas denuncias de grupos ambientalistas y del propio Ministerio del Ambiente de Brasil señalan una verdadera depredación de los recursos boscosos nativos para estos fines. En el año 2005, en declaraciones públicas, este propio organismo indicaba que el $80 \%$ del carbón vegetal que abastece las plantas siderürgicas en los estados de Marañón y Pará provenian de bosque nativo, lo que podria equivaler a una tala de 120 mil árboles por dia. En enero de 2006 se multó con 24 millones de reales (unos 5.500 millones de pesos) a dos siderúrgicas de Matto Grosso do Sul por irregularidades en el consumo de carbón vegetal. Últimamente, empresas brasileñas analizan inversiones en Bolivia para construir plantas de arrabio, las que se abastecerian de carbón vegetal procedente de bosques de ese pais.

En Brasil, las enormes magnitudes de la producción y consumo de carbón vegetal implican varios problemas asociados al recurso que deben ser corregidos. Como se indicó, el más significativo de ellos es el suministro de materia prima para la producción de carbón vegetal ya que se estima que cerca del $46 \%$ de la materia prima usada procede de los bosques nativos, mientras que las plantaciones estarian contribuyendo con el $36 \%$.

\section{Normas Internacionales}

Existen diferentes estándares o directrices que especifican los requerimientos que deben cumplir, tanto el carbón vegetal sólido como el carbón vegetal aglomerado (briquetas), y la manera de establecer determinados controles de calidad. Para el mercado global de Europa se aplica el estándar europeo EN 1860-2', en Alemania se utiliza la norma DIN 51749 que, de acuerdo a los exportadores internacionales, es la más exigente. El mercado de Suecia aplica la SS 187210, en tanto Bélgica emplea la NBM M 11-001 (Force Technology, 2008).

La Norma EN 1860-2 titulada "Carbón de barbacoas y briquetas de carbón de barbacoas, requerimientos y métodos de prueba" fue aprobada en octubre de 2004 por el Comité Europeo para Estandarización (CEN). Entre otros aspectos, la norma señala los requerimientos para el carbón sólido y el carbón briquetas en términos de aditivos no permitidos, muestras para análisis y métodos de testeo para determinar humedad, compuestos volátiles, cenizas, fijación de carbono y granulometria.

Algunos parámetros de esta norma son:

- Contenido máximo de humedad: $8 \%$.

- Fijación de carbono: Superior a $65 \%$.

- Sustancias fijadoras: Apropiadas para productos alimenticios.

- Envases: 5, 10, 15, 20 libras.

\footnotetext{
Norma creada por el CEN (European Committee for Standarization) cuyos miembros son: Austria, Bèlgica, Chipre. República Checa, Dinamarca, Estonia, Finlandia, Francia, Alemania, Grecia, Hungria, Islandia, Irlanda, Italia, Letonia, Luxemburgo, Malta. Holanda, Noruega, Polonia, Portugal, Eslovaquia, Eslovenia, España, Suecia, Suiza y Reino Unido
} 


\section{SITUACIÓN EN CHILE DEL CARBÓN VEGETAL}

Se calcula que la producción en Chile de carbón vegetal en 2006 fue de 251.000 t (FAO, 2008), cifra que no ha tenido grandes variaciones en los últimos años. Esta producción está destinada fundamentalmente al uso doméstico, principalmente en zonas rurales y en grupos familiares de escasos recursos. Esta cifra podria incluso ser mayor, considerando una importante porción de informalidad que caracteriza la comercialización de este producto. Se estima que 1 millón y medio de metros cúbicos anuales de madera (utilizando el factor $6 \mathrm{~m}^{3} \mathrm{ssc} / \mathrm{t}$ carbón (Basso y Quezada, 1970)) se destinarian a la producción de carbón vegetal en el país, estimando también que 44.000 ha de bosque nativo se intervienen anualmente para producir leña y carbón vegetal (Pacheco, 2005).

Existen pocos estudios respecto a la caracterización de la producción de carbón vegetal a nivel nacional y las disponibles normalmente se refieren a zonas o localidades muy acotadas. Sin embargo, en términos generales es posible señalar que el común denominador que gira en torno a la producción de carbón vegetal en el país es que está asociado a minifundistas de subsistencia, que dependen económicamente del recurso bosque, este último frecuentemente con avanzados signos de deterioro. Un estudio en la zona cercana a Linares concluyó que producir carbón vegetal tiene un costo muy similar a su precio de venta y agrega que es necesario abordar la problemática de la producción de carbón desde un punto de vista de rentabilidad social, cuestión que se agrava por la escasa presencia de alternativas laborales (Araya, 2003).

Es posible que dos personas trabajen en forma ininterrumpida, vale decir, dedicados cien por ciento a la actividad de carboneo, con dos hornos portátiles cada uno, en donde por cada horno se puede llegar a un nivel de ingresos de $\$ 600.000$ mensuales por concepto de venta total de la producción. A esta cifra se le deben descontar todos los gastos en que se incurre en la producción. Si bien, es cierto esta cantidad es muy difícil de lograr por parte del campesino por variados factores, como tener que dedicar tiempo a otras actividades del campo, da cuenta del potencial que presenta la venta de carbón y no de leña, en donde es posible aumentar el ingreso entre 2 a 3 veces por la venta de carbón, en relación al ingreso sólo obtenido por la venta de leña (Lobos, 2001).

Consideraciones respecto a la elaboración misma de carbón indican que es necesario orientar y capacitar a los productores nacionales en mejorar las técnicas de fabricación, como hornos con mejores rendimientos, humedad de la madera, especies, entre otros. En experiencias con hornos de barro, el rendimiento fluctúa entre $18 \%$ y $20 \%$, mientras que en Brasil, dónde se mejoró el manejo de las plantaciones y la leña utilizada entró más seca a los hornos, el rendimiento fue de un $26 \%$ (SERCOTEC, 1986, citado por Pacheco, 2005). Al respecto, la relación existente entre producción de leña verde y rendimiento de carbón está en proporción directa, perdiéndose cerca de un $70 \%$ del peso en el proceso de carbonización. En hornos de ladrillo tapizados de barro y paja, el rendimiento bordea el $20 \%$, en tanto en hornos de acero este valor podria ser bastante mayor. 


\section{Importaciones y Exportaciones de Carbón en Chile}

Chile, además de ser productor de carbón, se ha convertido en un importante importador de este producto. La importación de carbón vegetal a Chile ha crecido significativamente en los últimos años, desde poco más de 200 t que se importaban en 1995 a cerca de 26 mil que se alcanzó en el año 2007, es decir, ciento treinta veces más; la cantidad importada en este último año fue equivalente a 4,6 millones de dólares. Por el lado de las exportaciones, Chile prácticamente no realiza movimientos, siendo éstos marginales en cantidad y eventuales en el tiempo. En 2007 se exportaron apenas 63 t (INFOR, 2008).

La mayor parte del carbón importado por Chile proviene de Argentina, pais de larga tradición en producción y exportación de este producto. En 2006 Argentina exportó 151 mil toneladas de carbón vegetal, es decir, envió al exterior el $29 \%$ de su producción, en tanto que seis años atrás era el $17 \%$. Esto ha posicionado al pais trasandino como un importante proveedor mundial, cuyos principales mercados de destino en la actualidad son Europa, con el $73 \%$ de sus exportaciones, Chile con el $15 \%$ y Estados Unidos, con el 11\% (INFOR, 2008).

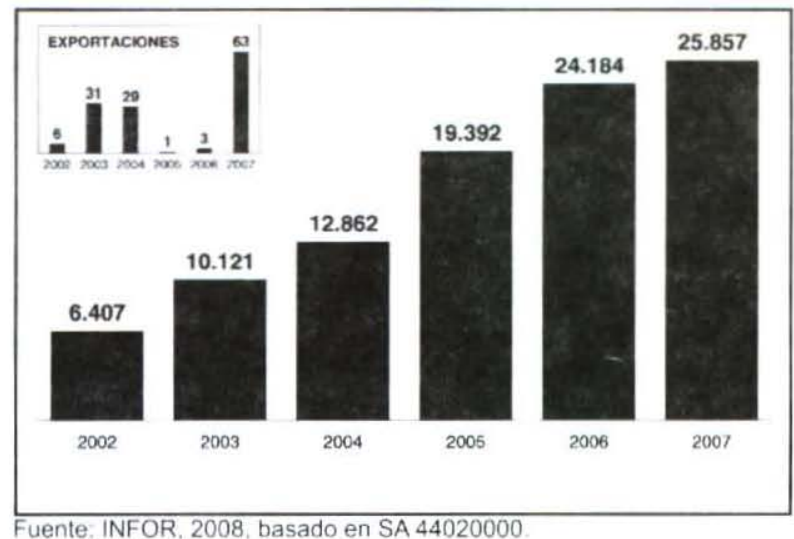

Figura $\mathrm{N}^{\circ} 7$

IMPORTACIONES DE CARBÓN VEGETAL EN CHILE (toneladas) 


\section{Cuadro $\mathrm{N}^{\circ} 4$ \\ IMPORTACIONES CHILENAS DE CARBÓN VEGETAL SEGÚN ORIGEN (toneladas)}

\begin{tabular}{|c|c|c|c|c|c|c|}
\hline País Origen & $\mathbf{2 0 0 2}$ & $\mathbf{2 0 0 3}$ & $\mathbf{2 0 0 4}$ & $\mathbf{2 0 0 5}$ & $\mathbf{2 0 0 6}$ & $\mathbf{2 0 0 7}$ \\
\hline Argentina & 5.876 & 9.361 & 12.007 & 17.467 & 21.899 & 21.980 \\
\hline Paraguay & 509 & 488 & 653 & 1.924 & 2.284 & 2.837 \\
\hline Ecuador & & & & & & 1.040 \\
\hline Brasil & & 259 & 195 & & & \\
\hline Otros & 22 & 13 & 7 & 1 & 1 & 0 \\
\hline Total & 6.407 & 10.121 & 12.862 & 19.392 & 24.184 & 25.857 \\
\hline
\end{tabular}

(Fuente: INFOR, 2008)

En las importaciones chilenas de carbón vegetal normalmente participan empresas dedicadas a la importación y distribución, generalmente de productos alimenticios y otros diversos, que tienen como sus principales clientes a grandes cadenas de supermercados o tiendas relacionadas en el pais. Este abastecimiento significa que el principal cliente consumidor de carbón vegetal es el dueño de casa, lo que sugiere que la demanda por parte del segmento de los clientes domiciliarios chilenos se ha ido incrementando en el tiempo. De esta forma, podría suponerse que el carbón vegetal importado a Chile se utiliza casi en un cien por ciento para el tradicional asado y no para otros usos, como sucede en Europa o Japón, donde una parte importante se emplea en la industria metalúrgica, para elaboración de carbón activado y otros usos.

Un antecedente adicional que complementa lo anterior es la estacionalidad en las internaciones de carbón vegetal, focalizándose éstas en el tercer y cuarto trimestre del año. Esto, evidentemente, tiene su origen en la mayor demanda que existe en fechas clave, como son las fiestas patrias y las celebraciones de fin de año, donde en promedio se importa entre 1,5 a 2 veces más carbón que en el resto del año. 


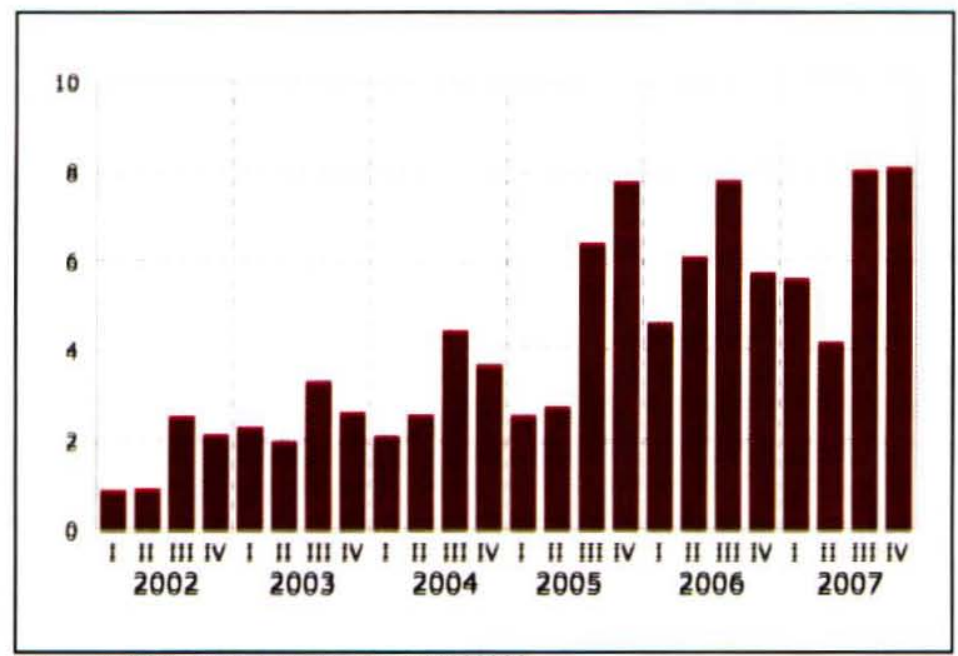

Fuente: INFOR, 2008, basado en SA 44020000.

Figura $\mathrm{N}^{\circ} 8$

IMPORTACIONES TRIMESTRALES DE CARBÓN VEGETAL EN CHILE (toneladas)

\section{Canales de Comercialización y Precios}

Los productores de carbón en Chile se caracterizan por su escaso poder de comercialización; su incapacidad de gestionar la venta de su producto y de llegar con éste al consumidor final. Generalmente el carbón es vendido a comerciantes intermediarios, que llegan con sus camiones a los predios en que ha sido producido o se encuentra guardado.

En su mayoria los productores no contactan a los intermediarios que comercializan el carbón en la ciudad. Esto puede incidir en los precios pagados en el campo, debido a que los productores se ven obligados a vender al precio que los intermediarios fijan. Muchos de los productores que contactan a los intermediarios, no logran acordar un precio adecuado para ellos, ya que no se observan diferencias en los precios de venta en comparación a los productores que no contactan al intermediario. Existe un porcentaje bajo de productores que participan en todo el proceso productivo (producción y comercialización); muy pocos productores tienen la capacidad de llegar al consumidor final, cosa que les permitiria obtener mayores utilidades por la venta de su carbón en los centros urbanos (Rodriguez, 2005).

En general, los canales de comercialización pueden ser: 1) Productor-consumidor, 2) Productor-camionero-consumidor, 3) Productor-camionero-local de venta-consumidor y 4) Productor-local de venta-consumidor. 


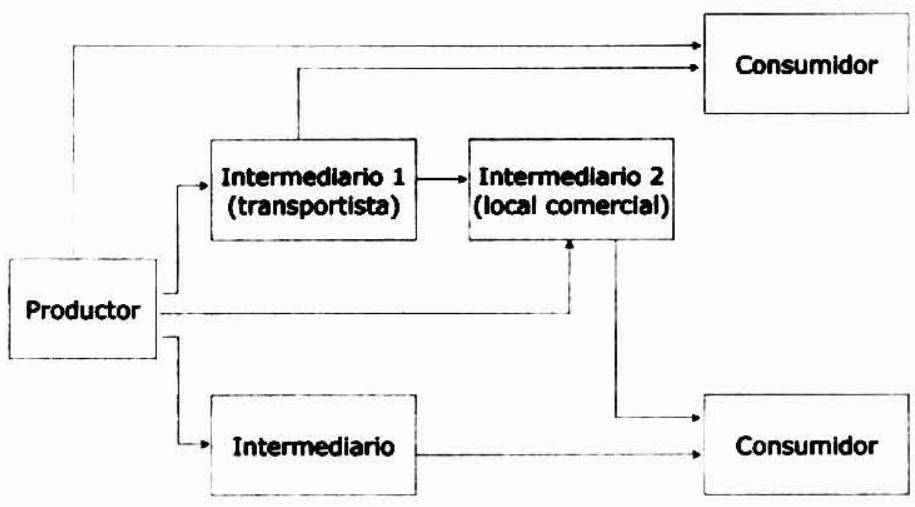

(Fuente: Rodriguez, 2005).

Figura $\mathrm{N}^{\circ} 9$

CANALES DE COMERCIALIZACIÓN DEL CARBÓN VEGETAL

En un estudio realizado en la zona de Chillán en el año 2005, se determinó que los precios de venta del carbón no tenian grades variaciones, situación que sería muy similar al resto del pais. El valor aproximado de un saco en origen puede variar entre $\$ 1.800$ y $\$ 2.200$.

\section{Cuadro $N^{\circ} 5$ \\ PRECIO DEL CARBÓN VEGETAL EN LA ZONA DE CHILLÁN \\ (Diciembre 2005)}

\begin{tabular}{|c|c|}
\hline Formato & Precio a público \\
\hline Saco $(18-22 \mathrm{~kg})$ & $\$ 3.000$ \\
\hline Bolsa plástica $(2,5 \mathrm{~kg})$ & $\$ 450$ \\
\hline Bolsa papel $(2,5 \mathrm{~kg})$ & $\$ 990$ \\
\hline
\end{tabular}

(Fuente: Rodriguez, 2005). 
En general, los precios de mercado son muy bajos, por lo que el margen es muy reducido o no alcanza a cubrir todos los costos reales de la producción de carbón. Por otro lado, no existe una diferenciación del producto en el mercado del carbón, por lo que el consumidor es sólo sensible al precio, en este sentido para subir el precio del producto es necesario diferenciarlo, a través de acciones de marketing. Dado que la rentabilidad del negocio depende mucho del tiempo dedicado por el productor a esta actividad, el propietario debe especializarse solamente en producir carbón vegetal, en mejorar su calidad y hacerlo a un menor costo.

Por el lado de las importaciones, el precio del carbón vegetal que ha llegado a Chile procedente del exterior promedió en el año 2007 US\$172 CIF/ t, lo que equivale en moneda nacional a unos $\$ 90^{2}$ el kilo. Por tipos de presentaciones, los más frecuentes son los sacos de entre 18 a 20 kilos y los sacos de 2,5 a 3 kilos. Estos últimos tienen entre un $10 \%$ a $13 \%$ más de precio que los sacos mayores, situación donde el costo del transporte juega un rol importante.

En promedio, el carbón nacional (espino) tiene mayor precio que el carbón importado. Sin embargo, esta diferencia posiblemente fue mayor en años anteriores y en la actualidad se aprecia escaso margen entre uno y otro precio.

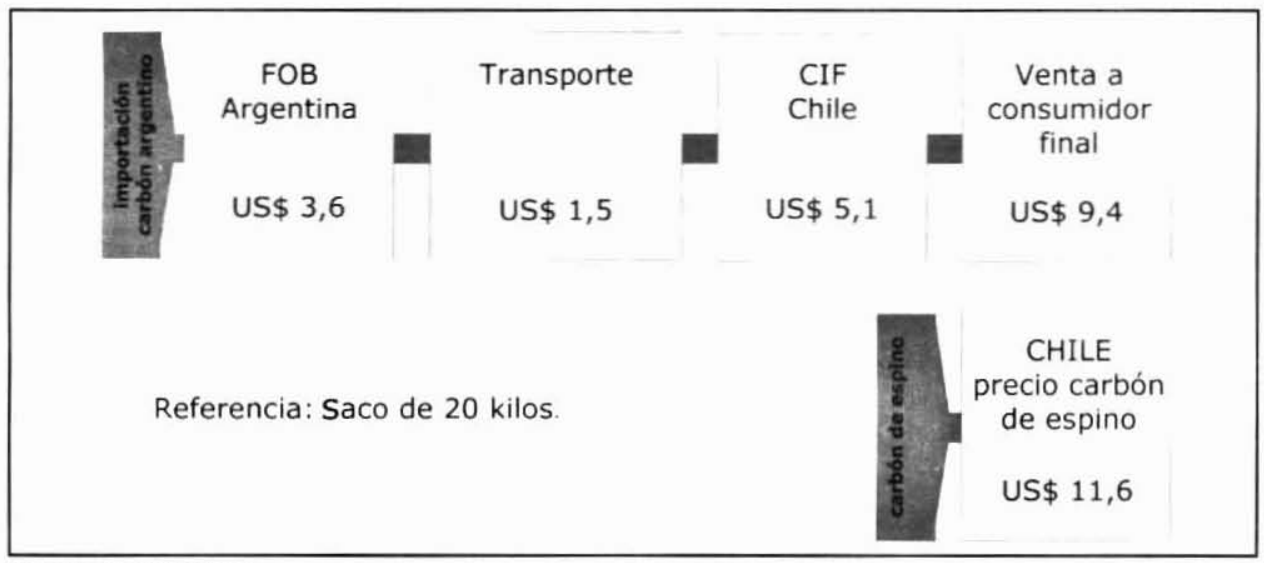

(Fuente: INFOR, 2008).

\section{Figura $\mathrm{N}^{\circ} 10$}

PRECIOS DE CARBÓN VEGETAL IMPORTADO DE ARGENTINA Y PRECIO DEL CARBÓN DE ESPINO CHILENO

(US\$/saco)

\section{Otros Antecedentes de Interés}

Para participar en el comercio internacional de carbón para consumo doméstico se debe cumplir con requisitos de orden técnico, de seguridad para el transporte, de packaging y, en el mercado europeo, se está incorporando progresivamente, el requisito de certificados de 
sustentabilidad de la producción. En primer lugar, el producto debe cumplir ciertas exigencias de calidad como, por ejemplo, contenido minimo de carbono fijo (normalmente se solicita la norma DIN alemana), porcentaje de humedad máximo tolerable y un rango del diámetro admitido para las particulas.

En el exterior, los consumidores prefieren que los pedazos de carbón sean de diámetro chico. Si bien los pedidos establecen rangos de 30 a $80 \mathrm{~mm}$, en la práctica se prefiere que no supere los $60 \mathrm{~mm}$ para los consumos domésticos. En las partidas destinadas a usos gastronómicos el tamaño de particulas es mayor, hasta los 120 mm o más. La norma DIN 51749 determina un contenido del $65 \%$ para las briquetas y del $75 \%$ para el carbón. No obstante, el contenido de carbono fijo del grueso de la oferta internacional de carbón se sitúa alrededor del $60 / 65 \%$.

También hay requisitos de seguridad para el transporte, por ser un producto combustible y auto inflamable; el carbón debe estar libre de polvillo y se debe realizar un análisis de la muestra en laboratorio para certificar que la partida no es auto inflamable, el contenido de humedad no debe superar el $7 \%$, y aun cuando se trata de exportaciones a granel, debe ir embolsado dentro del contenedor. También es obligatorio identificar el contenedor con un logo que indique que contiene un producto auto inflamable. Por la seguridad de los consumidores, en los paises desarrollados se prefiere el carbón que no chispea y se requiere que las bolsas estén cosidas en vez de abrochadas con ganchos metálicos.

Existen requisitos de packaging que difieren según el tipo de exportación, a granel o acondicionado para el consumidor final. En el primer caso, sólo se requiere que el carbón se almacene en bolsas de polipropileno dentro del contenedor. Cuando se trata de un producto listo para el consumo, aumentan los requisitos; en primer lugar, se debe fraccionar y envasar el producto con la marca y demás especificaciones que solicite el comprador, quien puede solicitar distintos tipos de envases, como por ejemplo un cierto tipo de bolsa o caja de cartón que contenga un determinado número de paquetes de 3,5 ó 10 kilogramos. Usualmente, estos paquetes se colocan sobre tarimas, listos para ser transportados a las bocas de expendio.

En el mercado europeo (uno de los principales destinos), existen requisitos de calidad para los envases que establecen el tipo del papel (usualmente no se utiliza kraft como en el mercado interno), la cantidad de pliegos de la bolsa, etc. Las bolsas deben estar cosidas con tres hilos, con una banda de papel crepé en la boca, para garantizar que no caiga polvillo. Además de las normas de envasado del producto en si, existen normas comunes al conjunto de los envases comerciales, que establecen que se debe utilizar papel fabricado a partir de pastas celulósicas libres de cloro y fijan la composición quimica de las tintas, que no deben contener pigmentos minerales. El incumplimiento de estas normas, que fueron establecidas por la Unión Europea para facilitar el reciclaje, implica una penalización económica para el comprador local. 


\section{CONCLUSIONES}

Si bien en Chile la producción de carbón vegetal no es nueva, un mejor desarrollo de este sector requiere de importantes esfuerzos en orientar y capacitar, especialmente a los pequeños propietarios productores involucrados, en mejorar los rendimientos y calidad del producto final de manera de ampliar la brecha entre el costo de producción y el precio de venta. Esfuerzos de marketing también son importantes.

Lo anterior ha sido posiblemente un factor clave en el aumento de las importaciones de carbón vegetal, el cual se compra principalmente a Argentina. Esta situación también refleja que el mercado interno chileno requiere cada vez más de este producto, derivado de una población con mayor ingreso per cápita y más demanda por tiempo libre y recreación.

Un escenario de desarrollo más inmediato del carbón vegetal en Chile debiera ser la exportación a Europa, orientándose a obtener un producto de mejorada calidad para el uso doméstico en ese mercado. En este continente existe una importante y creciente demanda por este producto, principalmente en Alemania, Bélgica e Italia.

Es necesario estudiar cada uno de estos paises en términos de sus requerimientos de carbón vegetal, como normas especificas, canales de comercialización, demanda, entre otros aspectos. De la misma forma, analizar el mercado japonés.

Estados Unidos no se perfila tan interesante en el corto o mediano plazo como destino de carbón vegetal, principalmente por el uso mayoritario de briquetas de carbón para el asado de alimentos más que de carbón sólido.

Es necesario profundizar en estudios del uso del carbón vegetal como combustible industrial, por ejemplo para generación de electricidad o industrias siderúrgicas, como sucede en Brasil. 


\section{REFERENCIAS}

Araya, F., 2003. "Caracterización de la producción de carbón en pequeños propietarios del sector Fundo Riquelme, Provincia Linares, VII Región". Tesis Universidad de Talca. Escuela de Ingenieria Forestal. Santiago, Chile. En <http://hdl.handle.net/1950/3663>

Basso, A.; Quezada, A., 1970. Tablas y factores de conversión. Manual № 7. Instituto Forestal. Santiago, Chile. 90 p.

Enciclopedia Encarta, 2008. Siderurgia. En: <http:/les.encarta.msn.com/ encyclopedia_761559225/Siderurgia.html>. Consulta: Mayo 2008.

FAO, 2008. Base de datos FAOSTAT de productos forestales de la FAO. En <http://faostat.fao. org/site/630/default.aspx>. Consulta: Mayo 2008.

Force Technology, 2008. Testing of barbecue coal and barbecue coal briquettes.

En <http://www.force.dk/en/Header/Download/Product+sheets/Inspection+Testing+and+Certifi cation/2705-1-en.htm> Consulta: Mayo 2008.

INFOR, 1987. Tablas de conversión mecánica y elaboración. Manual $\mathrm{N}^{\circ} 15$. INFOR-CORFO. Santiago Chile. $131 \mathrm{p}$.

INFOR, 2008. Base de datos de exportaciones de productos forestales. Instituto Forestal. Santiago, Chile.

Kuniko, 2001. Charcoal Adds to the Good Life. Nipponnia N¹9, Diciembre 2001.

En <http://web-japan.org/nipponia/nipponia19/en/topic/index.html> Consulta: Mayo 2008.

Lobos, M., 2001. Estudio preliminar sobre producción, comercialización y consumo de leña en la ciudad de Temuco. Documento $\mathrm{N}^{\circ} 3$. Serie de Publicaciones. WWF-CODEFF. Temuco, Chile. En <www.wwf.cl/publicaciones.htm>. Consulta: Mayo 2008.

Pacheco, G., 2005. Evaluación del proceso de carbonización y calidad del carbón de Acacia caven (Mol.) Mol., producido en hornos de barro. Tesis Universidad de Chile. Facultad de Ciencias Forestales. Santiago, Chile. 59 p.

Rodríguez, J., 2005. Estudio de rentabilidad económica de la producción de carbón proveniente de propietarios de bosque nativo de la precordillera de Ñuble. Proyecto Conservación y Manejo Sustentable del Bosque Nativo. Asociación Gremial por el Bosque Nativo de Ñuble, Chillán, 2005. 33 p.

En <www.chilebosquenativo.cl/info_documentos/comercializacion/Estudio $\% 20$ carb\%F3n $\% 20$ \%D1uble.pdf> Consulta: Mayo 2008.

SAGPyA, 2003. Producto: carbón vegetal. Informe exportaciones. Área de apoyo a los exportadores, Secretaria de Agricultura, Ganadería, Pesca y Alimentos. Buenos Aires-Argentina. $33 \mathrm{p}$.

En <www.sagpya.mecon.gov.ar/new/0-0/programas/apoyo/lnforme\%20Carbon\%20Vegetal. 
pdf > Consulta: Mayo 2008.

US Department of Agriculture, 2008. Foreing Agricultural Service's US Trade Internet System. US Department of Agriculture. En <http://www.fas.usda.gov/ffpd/fpd.html>. Consulta: Mayo 2008.

Waddams, C. 2000. Better energy services, better energy sectors-and links with the poors. Energy services for the world's poor, Energy and Development Report 2000. Energy Sector Management Assistance Programme, World Bank. En <http://www.esmap.org/pubs/index. asp>. Consulta: Mayo 2008. 